\title{
sciendo
}

\section{Effects of the Performance Level and Race Distance on Pacing in Ultra-Triathlons}

\author{
by \\ Beat Knechtle1,2, Caio Victor de Sousa3, Herbert Gustavo Simões ${ }^{3}$, \\ Thomas Rosemann ${ }^{2}$, Pantelis Theodoros Nikolaidis ${ }^{4}$
}

\begin{abstract}
The aim of this study was to examine the effects of the performance level and race distance on pacing in ultratriathlons (Double, Triple, Quintuple and Deca), wherein pacing is defined as the relative time (\%) spent in each discipline (swimming, cycling and running). All finishers ( $n=3,622)$ of Double, Triple, Quintuple and Deca Iron ultratriathlons between 1985 and 2016 were analysed and classified into quartile groups (Q1, Q2, Q3 and Q4) with Q1 being the fastest and $Q 4$ the slowest. Performance of all non-finishers $(n=1,000)$ during the same period was also examined. Triple and Quintuple triathlons (24.4\%) produced the highest rate of non-finishers, and Deca Iron ultra-triathlons produced the lowest rate $(18.0 \%)\left(\chi^{2}=12.1, p=0.007, \varphi_{c}=0.05\right)$. For the relative swimming and cycling times $(\%)$, Deca triathletes $(6.7 \pm 1.5 \%$ and $48.8 \pm 4.9 \%$, respectively) proved the fastest and Double $(9.2 \pm 1.6 \%$ and $49.6 \pm 3.6 \%)$ Iron ultra-triathletes were the slowest $(p<0.008)$ with $Q 4$ being the fastest group $(8.3 \pm 1.6 \%$ and $48.8 \pm 4.3 \%)$ and $Q 1$ the slowest one $(9.5 \pm 1.5 \%$ and $50.9 \pm 3.0 \%)(p<0.001)$. In running, Double triathletes were relatively the fastest $(41.2$ $\pm 4.0 \%)$ and Deca $(44.5 \pm 5.4 \%)$ Iron ultra-triathletes the slowest $(p<0.001)$ with $Q 1$ being the fastest $(39.6 \pm 3.3 \%)$ and $Q 4$ the slowest group $(42.9 \pm 4.7 \%)(p<0.001)$. Based on these findings, it was concluded that the fastest ultratriathletes spent relatively more time swimming and cycling and less time running, highlighting the importance of the role of the latter discipline for the overall ultra-triathlon performance. Furthermore, coaches and ultra-triathletes should be aware of differences in pacing between Double, Triple, Quintuple and Deca Iron triathlons.
\end{abstract}

Key words: swimming, cycling, running, ultra-endurance, performance.

\section{Introduction}

Little is known about pacing in longdistance triathlons such as the Ironman distance triathlon (i.e. $3.8 \mathrm{~km}$ swimming, $180 \mathrm{~km}$ cycling and $42.195 \mathrm{~km}$ running). Pacing in triathlons can be considered within each discipline (i.e. swimming, cycling and running) or how the athlete distributes energy across the disciplines. Elite male and female Ironman triathletes tend to adopt a positive pacing strategy (i.e. speed decreases throughout a race) in both the running and the cycling splits (Angehrn et al., 2016). Also, or longer triathlon race distances such as a multi-stage Ironman triathlon covering ten times the Ironman distance within ten days, performance progressively declined over the days (Herbst et al., 2011; Knechtle et al., 2014).

In that regard, a Double Iron ultratriathlon covers $7.6 \mathrm{~km}$ of swimming, $360 \mathrm{~km}$ of cycling and $84.4 \mathrm{~km}$ of running, a Triple Iron ultratriathlon covers $11.4 \mathrm{~km}$ of swimming, $540 \mathrm{~km}$ of cycling and $126.6 \mathrm{~km}$ of running, a Quintuple Iron ultra-triathlon covers $19 \mathrm{~km}$ of swimming, $900 \mathrm{~km}$ of cycling and $211 \mathrm{~km}$ of running and a Deca Iron ultra-triathlon covers $38 \mathrm{~km}$ of swimming, $1800 \mathrm{~km}$ of cycling and $422 \mathrm{~km}$ of running (Knechtle et al.,

1 - Gesundheitszentrum St. Gallen, St. Gallen, Switzerland.

2 - Institute of Primary Care, University of Zurich, Zurich, Switzerland.

3 - Graduate Program in Physical Education, Catholic University of Brasilia, DF, Brazil.

4 - Exercise Physiology Laboratory, Nikaia, Greece. 
2011; Lepers et al., 2011). An athlete who competes in a race of such a length should know the significance of each discipline (swimming, cycling or running) in order to best strategize his/her pacing.

In endurance and ultra-endurance races, pacing is crucial for a successful finish (Abbiss and Laursen, 2008; Foster et al., 1993, 2005). Pacing can be defined as the strategy by which effort is managed throughout an exercise session in relation to a specific goal and with the knowledge of the likely demands of the task (Edwards and Polman, 2012); or, alternatively, as the the process by which the total energy expenditure during exercise is regulated on a moment-to-moment basis in order to ensure that the exercise session can be completed within a minimum time and without catastrophic biological failure (Baron et al., 2011). According to Abbiss and Laursen (2008), six different strategies in pacing can be distinguished, such as negative pacing (speed increases during a race), positive pacing (speed decreases), all-out pacing (maximal effort from the start), even pacing (constant speed), parabolic-shaped pacing (speed decreases in the first part and increases in the last part of a race) and variable pacing (speed changes due to environmental conditions).

In sprint and short distance triathlons, performance in split disciplines has an effect on overall race performance (Le Meur et al., 2011; Vleck et al., 2006; Wu et al., 2015, 2016). In a sprint distance triathlon, positive pacing in swimming leads to faster cycling and overall race times ( $\mathrm{Wu}$ et al., 2016). In an Olympic distance triathlon, a reduction in initial running speed (Le Meur et al., 2011) and a decrease in cycling speed with an increase in speed variablity (Bernard et al., 2009) leading to a more even pacing seemed to be associated with improved overall race time.

Additionally, sex has an influence on pacing during a triathlon race (Le Meur et al., 2009; Vleck et al., 2008). In an Olympic distance triathlon, male and female elite triathletes adopted similar positive pacing strategies during both the swimming and the running split. Female triathletes, however, showed differences during the cycling and running splits compared to male athletes (Le Meur et al., 2009).

Although data on pacing in cycling and running splits among elite Ironman triathletes exists (Angehrn et al., 2016), as well as for the contribution of swimming and cycling splits to overall Ironman triathlon race time (Figueiredo et al., 2016), we have no data on pacing and the contribution of each discipline in ultra-triathlons. Such knowledge would be of both theoretical and practical importance to sports scientists and coaches, respectively. Ultra-triathletes may participate in Iron ultra-triathlons of various distances; consequently, knowing whether pacing varies with distance might help them to adjust their pacing strategies accordingly. Furthermore, profiling the relative contribution of swimming, cycling and running to the overall race time by the performance level may guide triathletes to select optimal pacing strategies, depending on their competition goal.

A further consideration is that triathletes competing in long-distance races may have a background in swimming, cycling or running. Thus, athletes with a strong background in a specific discipline want to know whether their 'strong' discipline enables them to finish the race among the best and/or whether they need to pay attention to their 'weak' discipline in order to avoid dropping out due to over pacing in their 'strong' discipline or losing too much time in the 'weak' discipline.

Therefore, the aim of the present study was to examine the variation of pacing within four versions of ultra-triathlons (i.e. Double, Triple, Quintuple and Deca Iron ultra-triathlon) as well as the role of the performance level. We analysed data from all ultra-triathlon races held worldwide between 1985 and 2016, and adopted the hypotheses that the relatively fastest swimmers were not the fastest finishers, the fastest cyclists were among the fastest finishers, the fastest runners were the fastest finishers, and that these statements would be true for both women and men. Moreover, the secondary aim was to study the competition density, the variation in participation and distribution of finishers/nonfinishers by sex and version of Iron ultra-triathlon.

\section{Methods}

\section{Ethics approval}

This study was approved by the Institutional Review Board of Kanton St. Gallen, Switzerland, with a waiver of the requirement of informed consent by the participants as the study involved the analysis of publicly available data. 


\section{Methodology}

Data from all male and female starters competing in all ultra-triathlon races held since 1985 until the end of 2008 was obtained from the race directors and collected by one of the authors. Data from 2009 to 2016 is publicly available at the website of the International Ultra-Triathlon Association (IUTA): www.iutasport.com/archives/results.php. The initial data set consisted of 4,622 participants; among them 3,622 were finishers and 1,000 nonfinishers. Very few Quadruple and Double Deca Iron ultra-triathlons have been organized since 1985, and these races were not held on a regular basis in contrast to the other race distances, so they were not included in our analysis.

\section{Statistical analysis}

All statistical analyses were performed using the statistical package IBM SPSS v.20.0 (SPSS, Chicago, USA). Data was presented as means \pm standard deviation. We examined the association of finishers/non-finishers between sex and each analysed version of Iron ultra-triathlons using chi-square $\left(\chi^{2}\right)$ and Cramer's phi $(\varphi c)$ to evaluate the magnitude $(\varphi c<0.10$, trivial; $0.10 \leq \varphi \mathrm{c}$ $<0.30$, small; $0.30 \leq \varphi c<0.50$, medium; $\varphi c \geq 0.50$, large) of this association. Swimming, cycling and running split times were calculated as the percentage of the overall race time using the formula: 100×discipline time/race time. Performance groups were calculated as quartiles of the overall race time: Q1 (the fastest), Q2, Q3 and Q4 (the slowest). A between-within subjects analysis of variance (ANOVA) examined the main effects of the performance level, distance version and their interaction on split times as the percentage of the total race time. Each sex was examined separately. The Bonferroni post-hoc multiple comparison test showed differences among the groups. A two-way ANOVA examined the relationship between finishers/non-finishers with regard to swimming and cycling speed. The relationship between finishers/non-finishers and running speed was not considered since it was not possible to calculate the relative running time for non-finishers. The magnitude of differences in the ANOVAs was evaluated using eta squared $\left(\eta^{2}\right)$ and categorized as trivial $\left(\eta^{2}<0.01\right)$, small $\left(0.01 \leq \eta^{2}<0.06\right)$, moderate $\left(0.06 \leq \eta^{2}<0.14\right)$ and large $\left(\eta^{2} \geq 0.14\right)$. To study differences in swimming, cycling and running split times expressed as the proportion (\%) of the total race time for different distances of the Iron ultra-triathlon and performance levels (quartiles), we used a mixedeffects regression model with triathletes as a random variable, whereas the distance version and performance level were assigned as fixed variables. In addition, we examined interaction effects between these fixed variables. The Akaike information criterion (AIC) was used to select the final model. Competition density (CD) was calculated as competitors per unit of finish time (competitors/min) for each sex and distance version. The Alpha level was set at 0.05 .

\section{Results}

Association of finishers/non-finishers to the version of Iron ultra-triathlon and sex

Among all of the participants, we observed a trivial association between finishers/nonfinishers and the versions of Iron ultra-triathlons $\left(\chi^{2}=12.1, p=0.007, \varphi c=0.05\right)$, with the percentage of non-finishers being 20.2, 24.4, 24.4 and $18.0 \%$ in the Double, Triple, Quintuple and Deca Iron ultratriathlon, respectively (Figure 1). The fact of finishing the race was not associated with sex $\left(\chi^{2}=\right.$ $1.7, p=0.192, \varphi c=-0.02)$, i.e. a similar percentage of non-finishers was observed for both women and men (24.0 and $21.4 \%$, respectively). For women, a small association between finishers/non-finishers and the particular version of Iron ultra-triathlons $\left(\chi^{2}=9.8, p=0.021, \varphi c=0.14\right)$ was found, wherein the percentage of non-finishers were 24.9, 18.1, 33.3 and $18.1 \%$ in the Double, Triple, Quintuple and Deca Iron ultra-triathlon, respectively. This association was also observed for men, yet with trivial magnitude $\left(\chi^{2}=20.0, p<0.001, \varphi c=0.07\right)$, and respective percentages of $19.6,25.0,22.5$ and $14.5 \%$. Effect of the version of the Iron ultra-triathlon and performance level on swimming time

All data in absolute values is presented in Table 1. Among female finishers, a moderate effect of the distance version on swimming time (\%) was observed $\left(p<0.001, \eta^{2}=0.091\right)$. The Deca Iron ultratriathletes were relatively the fastest, while the Double Iron ultra-triathletes the slowest (Figure 2, Table 2). A small effect of the performance level was shown $\left(p=0.004, \eta^{2}=0.037\right)$, where Q1 was relatively the slowest and Q4 the fastest. No distance version $\times$ performance level interaction was found $\left(p=0.184, \eta^{2}=0.035\right)$. For men, a moderate effect of the distance version on 
swimming time $(\%)$ was observed $\left(p<0.001, \eta^{2}=\right.$ 0.136), where the Deca Iron ultra-triathletes were relatively the fastest and the Double Iron ultratriathletes the slowest. A small effect of the performance level was shown $\left(p<0.004, \eta^{2}=0.019\right)$, where Q1 was relatively the slowest and Q4 the fastest. No distance version $\times$ performance level interaction was found $\left(p=0.578, \eta^{2}=0.002\right)$.

Table 1

Splits and overall absolute performance of female and male finishers in ultra-triathlon events. Data expressed as mean and ( \pm ) standard deviation.

\begin{tabular}{|c|c|c|c|c|c|}
\hline & & $\begin{array}{l}\text { Swim } \\
\text { (min) }\end{array}$ & $\begin{array}{l}\text { Cycle } \\
\text { (min) }\end{array}$ & $\begin{array}{l}\text { Run } \\
\text { (min) }\end{array}$ & $\begin{array}{c}\text { Overall } \\
(\mathrm{min})\end{array}$ \\
\hline \multirow[t]{2}{*}{ Double Iron } & Women & $166.5 \pm 30.2$ & $925.5 \pm 124.4$ & $747.9 \pm 146.3$ & $1852.0 \pm 257.7$ \\
\hline & Men & $157.0 \pm 31.5$ & $852.2 \pm 128.5$ & $714.0 \pm 139.3$ & $1730.1 \pm 250.4$ \\
\hline \multirow[t]{2}{*}{ Triple Iron } & Women & $250.6 \pm 30.8$ & $1497.7 \pm 215.1$ & $1226.6 \pm 262.7$ & $2989.6 \pm 438.9$ \\
\hline & Men & $240.1 \pm 40.8$ & $1404.8 \pm 211.1$ & $1221.9 \pm 246.8$ & $2875.9 \pm 412.5$ \\
\hline \multirow[t]{2}{*}{ Quintuple Iron } & Women & $576.4 \pm 153.6$ & $3685.1 \pm 837.3$ & $3047.5 \pm 556.2$ & $7365.7 \pm 1346.7$ \\
\hline & Men & $479.8 \pm 87.2$ & $3176.9 \pm 674.1$ & $2820.4 \pm 651.7$ & $6540.9 \pm 1187.7$ \\
\hline \multirow[t]{2}{*}{ Deca Iron } & Women & $1205.1 \pm 134.5$ & $9109.6 \pm 1814.9$ & $7273.8 \pm 563.8$ & $17588.9 \pm 1876.4$ \\
\hline & Men & $1063.7 \pm 265.1$ & $7814.8 \pm 1436.1$ & $7211.5 \pm 1559.8$ & $16105.8 \pm 2599.4$ \\
\hline
\end{tabular}

Table 2

Coefficients (C) and standard errors of estimate (SEE) from multi-variate regression models for the swimming performance (\%) by a distance version and the performance level in women and men.

\begin{tabular}{|c|c|c|c|c|c|c|}
\hline \multirow[b]{2}{*}{ Variable } & \multicolumn{3}{|c|}{ Women } & \multicolumn{3}{|c|}{ Men } \\
\hline & C & SEE & $p$ & C & SEE & $p$ \\
\hline Intercept & 9.07 & 0.35 & $<0.001$ & 9.43 & 0.12 & $<0.001$ \\
\hline Distance version & -0.59 & 0.21 & 0.007 & -0.77 & 0.07 & $<0.001$ \\
\hline [Performance level=1] & 0.98 & 0.49 & 0.045 & 1.07 & 0.16 & $<0.001$ \\
\hline [Performance level =2] & 1.23 & 0.49 & 0.012 & 0.60 & 0.16 & $<0.001$ \\
\hline [Performance level =3] & 0.60 & 0.49 & 0.217 & 0.55 & 0.16 & 0.001 \\
\hline [Performance level =4] & $0^{*}$ & $0^{*}$ & . & $0^{*}$ & $0^{*}$ & . \\
\hline $\begin{array}{l}{[\text { Performance level }=1] \times \text { Distance }} \\
\text { version }\end{array}$ & 0.34 & 0.30 & 0.261 & 0.05 & 0.10 & 0.623 \\
\hline $\begin{array}{l}{[\text { Performance level }=2] \times \text { Distance }} \\
\text { version }\end{array}$ & -0.38 & 0.30 & 0.216 & -0.01 & 0.10 & 0.882 \\
\hline $\begin{array}{l}{[\text { Performance level }=3] \times \text { Distance }} \\
\text { version }\end{array}$ & -0.17 & 0.30 & 0.581 & -0.13 & 0.10 & 0.191 \\
\hline $\begin{array}{l}{[\text { Performance level }=4] \times \text { Distance }} \\
\text { version }\end{array}$ & $0^{*}$ & $0^{*}$ & . & $0^{*}$ & $0^{*}$ & $\cdot$ \\
\hline
\end{tabular}

${ }^{*}$ This variable was set to zero because it was redundant. 
Table 3

Coefficients (C) and standard errors of estimate (SEE) from multi-variate regression models for the cycling performance (\%) by a distance version and the performance level in women and men.

\begin{tabular}{|c|c|c|c|c|c|c|}
\hline & \multicolumn{3}{|c|}{ Women } & \multicolumn{3}{|c|}{ Men } \\
\hline Variable & C & SEE & $p$ & $\mathrm{C}$ & SEE & $p$ \\
\hline Intercept & 47.62 & 0.82 & $<0.001$ & 49.15 & 0.30 & $<0.001$ \\
\hline Distance version & 1.33 & 0.51 & 0.010 & -0.30 & 0.18 & 0.101 \\
\hline [Performance level=1] & 5.15 & 1.16 & $<0.001$ & 1.99 & 0.42 & $<0.001$ \\
\hline [Performance level =2] & 4.10 & 1.16 & $<0.001$ & 0.81 & 0.42 & 0.056 \\
\hline [Performance level =3] & 1.55 & 1.15 & 0.178 & 0.07 & 0.42 & 0.866 \\
\hline [Performance level =4] & $0^{*}$ & $0^{*}$ & . & $0^{*}$ & $0^{*}$ & . \\
\hline $\begin{array}{l}{[\text { Performance level }=1] \times \text { Distance }} \\
\text { version }\end{array}$ & -2.08 & 0.72 & 0.004 & 0.11 & 0.26 & 0.671 \\
\hline $\begin{array}{l}{[\text { Performance level }=2] \times \text { Distance }} \\
\text { version }\end{array}$ & -1.94 & 0.72 & 0.007 & -0.13 & 0.26 & 0.608 \\
\hline $\begin{array}{l}{[\text { Performance level }=3] \times \text { Distance }} \\
\text { version }\end{array}$ & -0.88 & 0.72 & 0.221 & -0.09 & 0.26 & 0.714 \\
\hline $\begin{array}{l}{[\text { Performance level }=4] \times \text { Distance }} \\
\text { version }\end{array}$ & $0^{*}$ & $0^{*}$ & . & $0^{*}$ & $0^{*}$ & . \\
\hline
\end{tabular}

${ }^{*}$ This variable was set to zero because it was redundant.

Table 4

Coefficients (C) and standard errors of estimate (SEE) from multi-variate regression models for the running performance (\%) by a distance version and the performance level in women and men.

\begin{tabular}{|c|c|c|c|c|c|c|}
\hline & \multicolumn{3}{|c|}{ Women } & \multicolumn{3}{|c|}{ Men } \\
\hline Variable & C & SEE & $\mathrm{p}$ & C & SEE & $\mathrm{p}$ \\
\hline Intercept & 43.30 & 0.89 & $<0.001$ & 41.42 & 0.33 & $<0.001$ \\
\hline Distance version & -0.74 & 0.56 & 0.184 & 1.07 & 0.20 & $<0.001$ \\
\hline [Performance level=1] & -6.13 & 1.27 & $<0.001$ & -3.06 & 0.47 & $<0.001$ \\
\hline [Performance level =2] & -5.33 & 1.27 & $<0.001$ & -1.41 & 0.47 & 0.002 \\
\hline [Performance level =3] & -2.16 & 1.26 & 0.087 & -0.62 & 0.47 & 0.185 \\
\hline$[$ Performance level $=4]$ & $0^{*}$ & $0^{*}$ & 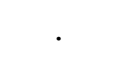 & $0^{*}$ & $0^{*}$ & 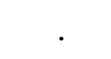 \\
\hline $\begin{array}{l}{[\text { Performance level }=1] \times} \\
\text { Distance version }\end{array}$ & 1.74 & 0.79 & 0.028 & -0.16 & 0.28 & 0.574 \\
\hline $\begin{array}{l}{[\text { Performance level }=2] \times} \\
\text { Distance version }\end{array}$ & 2.31 & 0.78 & 0.003 & 0.15 & 0.28 & 0.602 \\
\hline $\begin{array}{l}{[\text { Performance level }=3] \times} \\
\text { Distance version } \\
{[\text { Performance level }=4] \times} \\
\text { Distance version }\end{array}$ & $\begin{array}{c}1.05 \\
0^{*}\end{array}$ & $\begin{array}{c}0.78 \\
0^{*}\end{array}$ & 0.182 & $\begin{array}{c}0.23 \\
0^{*}\end{array}$ & $\begin{array}{c}0.28 \\
0^{*}\end{array}$ & 0.425 \\
\hline
\end{tabular}

*This variable was set to zero because it was redundant. 

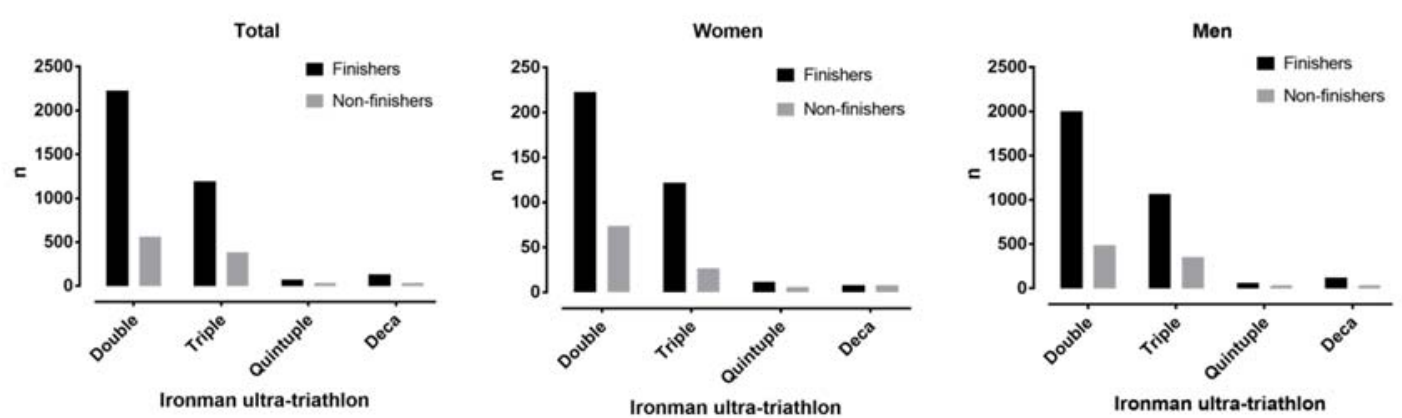

Figure 1

Distribution of finishers and non-finishers by the version of Iron ultra-triathlons in total, women and men.
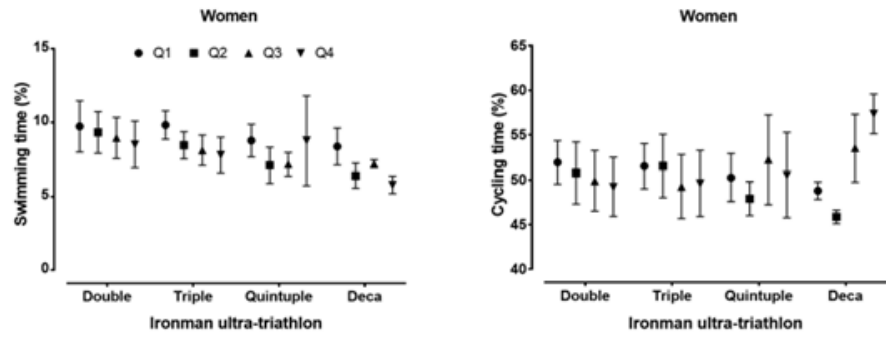

Men


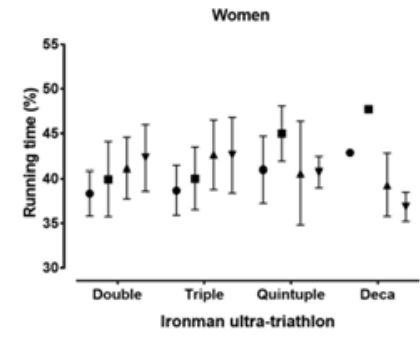

Men

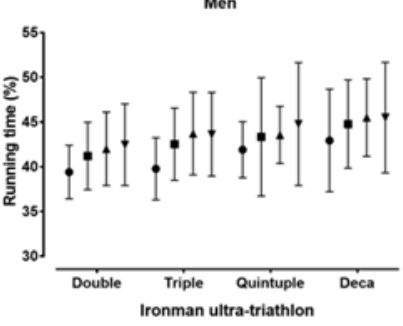

Figure 2

Swimming, cycling and running time in proportion (\%) of the total race time by the performance level and the version of Iron ultra-triathlons in women and men. 

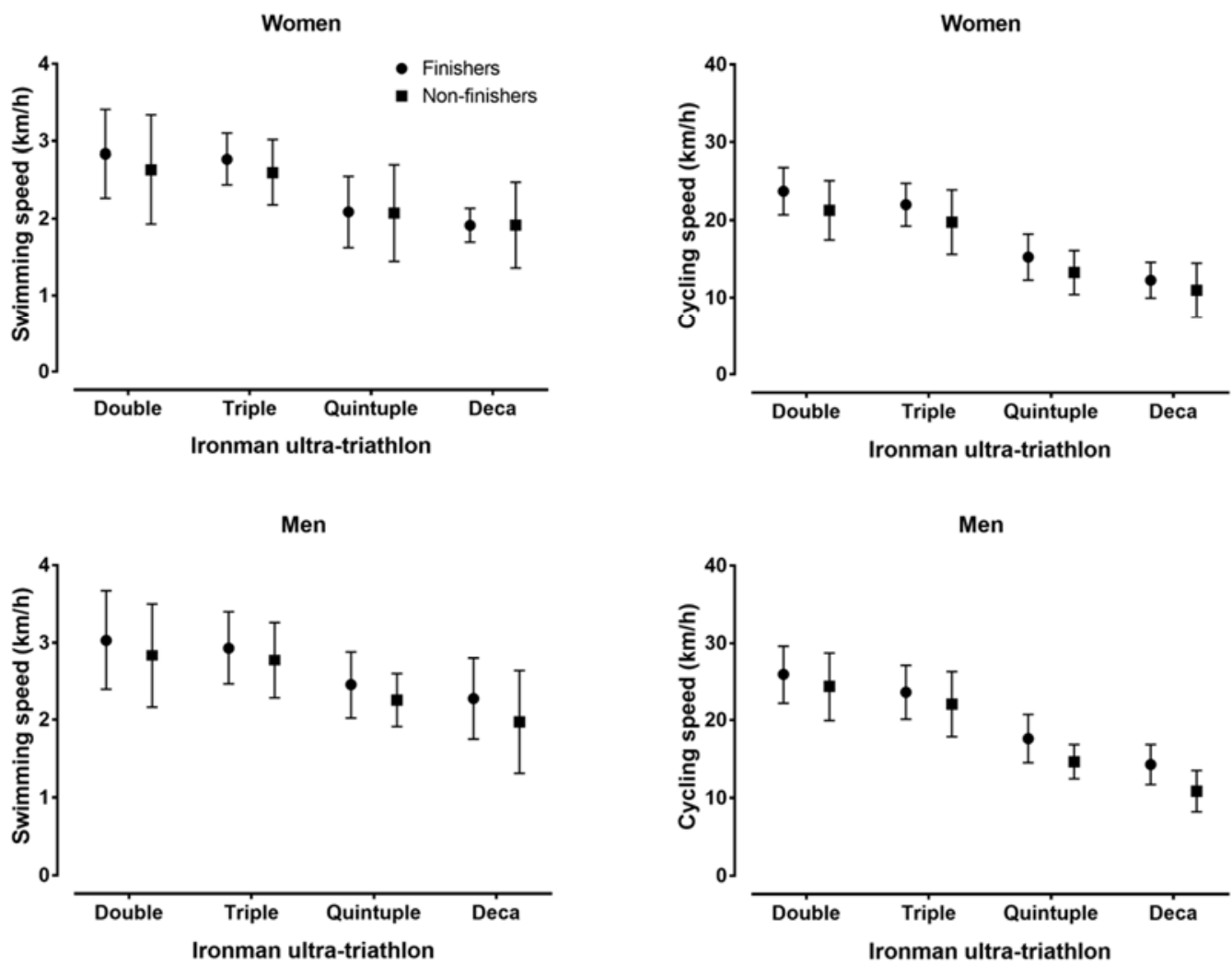

Figure 3

Swimming and cycling speed in finishers and non-finishers by the version of Iron ultratriathlons.

Effect of the version of the Iron ultra-triathlon and the performance level on cycling time

With regard to cycling time (\%) among women, no effects were observed either for the distance version $\left(p=0.869, \eta^{2}=0.002\right)$ or for the performance level $\left(p=0.074, \eta^{2}=0.020\right)$, however, a moderate distance version $\times$ performance level interaction was shown $\left(p<0.001, \eta^{2}=0.075\right)$ (Table $3)$. For men, a trivial effect of the distance version on cycling time expressed as relative to overall race time $(\%)$ was observed $\left(p=0.003, \eta^{2}=0.004\right)$, where the Deca Iron ultra-triathletes were relatively the fastest and the Double Iron ultra-triathletes the slowest. A trivial effect of the performance level on cycling time was shown $\left(p<0.001, \eta^{2}=0.007\right)$, where
Q1 was relatively the slowest and Q4 the fastest. No distance version $\times$ performance level interaction was found $\left(p=0.347, \eta^{2}=0.003\right)$.

Effect of the version of the Iron ultra-triathlon and the performance level on running time

For women, no main effect of the distance version on running time $(\%)$ was observed $(p=$ 0.277, $\left.\eta^{2}=0.011\right)$ nor a main effect of the performance level on running time $\left(p=0.057, \eta^{2}=\right.$ 0.021 ). A moderate distance version $\times$ performance level interaction was shown $\left(p=0.003, \eta^{2}=0.069\right)$ (Table 4). For men, a small effect of the distance version on running time $(\%)$ was observed $(p<$ $\left.0.001, \eta^{2}=0.038\right)$, where the Double Iron ultra-triathletes were relatively the fastest and 
the Deca Iron ultra-triathletes the slowest. A small effect of the performance level was shown $(p<$ $\left.0.001, \eta^{2}=0.016\right)$, where $\mathrm{Q} 4$ was relatively the slowest and Q1 the fastest. No distance version $\times$ performance level interaction was found $(p=0.248$, $\left.\eta^{2}=0.004\right)$. In summary, compared to swimming and cycling, we observed an opposite effect of the distance version and performance level on running time (\% of total race time) for men.

Swimming and cycling speed in finishers and nonfinishers

Comparing finishers and non-finishers for their swimming speed $(\mathrm{km} / \mathrm{h})$, no relationship between the finisher/non-finisher and speed was observed $\left(p=0.352, \eta^{2}=0.002\right)$ (Figure 3$)$. No distance version $\times$ finisher/non-finisher interaction was shown $\left(p=0.828, \eta^{2}=0.002\right)$. For men, a trivial relationship between the finisher/non-finisher and swimming speed was found $\left(p<0.001, \eta^{2}=0.004\right)$, where finishers were relatively faster than nonfinishers. No distance version $\times$ finisher/nonfinisher interaction was shown $\left(p=0.764, \eta^{2}<\right.$ 0.001).

In cycling speed $(\mathrm{km} / \mathrm{h})$, an interaction between the finisher/non-finisher and speed was found among women $\left(p=0.004, \eta^{2}=0.020\right.$, small magnitude) and among men $\left(p<0.001, \eta^{2}=0.009\right.$, trivial magnitude), where finishers were relatively faster than non-finishers. No distance version $x$ finisher/non-finisher interaction was shown either for women $\left(p=0.921, \eta^{2}=0.001\right)$ or for men $(p=$ $0.225, \eta^{2}=0.001$ ).

\section{Competition density}

Competition density (CD) for women was $0.176,0.033,0.002$ and 0.002 competitors/min in the Double, Triple, Quintuple and Deca Iron ultratriathlons, whereas the respective values for men were $1.069,0.501,0.010$ and 0.010 competitors $/ \mathrm{min}$. Thus, CD was higher for men than for women, and it decreased with the version's distance.

\section{Discussion}

Based upon findings for shorter triathlon distance races, we hypothesized that for ultratriathletes, the relatively fastest swimmers were not the fastest finishers, the fastest cyclists were among the fastest finishers, and the fastest runners were the fastest finishers. The main findings of the present study were that (i) running was relatively the fastest in the Double Iron ultra-triathlon and the slowest in the Deca Iron ultra-triathlon with Q1 being relatively the fastest and Q4 the slowest, (ii) swimming was relatively the fastest in the Deca Iron ultra-triathlon and the slowest in the Double Iron ultra-triathlon with Q4 (the overall slowest group) being relatively the fastest and Q1 the slowest, (iii) the same trends as in swimming, but with weaker magnitude were observed for cycling, (iv) the highest rate of non-finishers was found in the Triple and Quintuple and the lowest in the Deca Iron ultra-triathlon, $(v)$ very small differences in swimming and cycling speed were observed between finishers and non-finishers, and (vi) competitor density was higher for men and in the short distance versions. Regarding our hypothesis, we found differences for shorter ultra-triathlon races (i.e. Double Iron ultra-triathlon) where the fastest runners were the fastest finishers and longer ultra-triathlon races (i.e. Quintuple and Deca Iron ultra-triathlon) where the fastest swimmers and cyclists were the fastest finishers.

Higher proportion of finishers in the longest race distance

Regarding the prevalence of non-finishers: the highest rate was found in the Triple and Quintuple Iron ultra-triathlon and the lowest in the Deca Iron ultra-triathlon. The Deca Iron ultratriathlon might be considered more demanding compared to the shorter Iron ultra-triathlon versions and one would expect higher rates of nonfinishers. This observation might be partially attributed to the relatively small number of participants in the Deca Iron ultra-triathlon compared to the more popular short distances (Knechtle et al., 2011).

The relatively fastest swimmers were the slowest in overall race performance

The relatively fastest swimming times were achieved in the Deca Iron ultra-triathlon, whereas athletes in the Double Iron ultra-triathlon spent a greater relative proportion of race time while swimming (i.e. they were relatively slower). In addition, the fastest performance group was relatively the slowest in swimming for all versions of ultra-triathlons. It is well known that the contribution of swimming to overall ultra-triathlon performance is lower than those of cycling and running (Lepers et al., 2011). In a Triple Iron ultratriathlon, performance in the swimming split seemed to be of lesser importance compared to the cycling and running splits (Knechtle and Kohler, 2009). 
Therefore, the present findings are different to findings for shorter triathlon distances. In sprint and Olympic distance triathlons, swimming pace may dictate whether the athlete will be in the leading cycling group (fastest) or a chase group (slowest) suggesting that swimming performance is of great importance for overall race performance (Peeling and Landers, 2009). In a sprint distance triathlon, positive pacing in swimming leads to faster cycling and overall race times (Wu et al., 2016). However, in sprint and Olympic distance triathlons, swimming at a reduced intensity seemed to enhance performance in the subsequent disciplines (Peeling and Landers, 2009). Peeling and Landers (2009) suggested that conserving enough energy might be an optimal swimming pace strategy for shorter distances in order to ensure that the athlete would remain in the fastest "pack".

On the other hand, a slower swimming speed might be a tactical decision. In the Olympic distance triathlon, slower swimming might lead to greater work in the beginning of the cycling split, which influences the running split (Vleck et al., 2006). Similarly, slower swimming leads to faster cycling and consequently to a faster overall race time (Peeling et al., 2005). A potential reason for an improved cycling performance after the swimming split could also be the influence of drafting. It has been shown that cycling efficiency significantly improved when the cycling session was preceded by a swim performed in a drafting position rather than an isolated swim (Delextrat et al., 2003).

The triathletes that had the fastest cycling time had the slowest overall race performance

The third main finding was that cycling splits showed similar results to swimming splits, but with smaller magnitude, where the Deca Iron ultra-triathlons showed relatively the fastest swimming and cycling times and the Double Iron ultra-triathlons had the slowest swimming times. Moreover, the fastest performance group was relatively the slowest in cycling.

A fundamental aspect that differentiates between Olympic and sprint distance triathlons and an ultra-triathlon is drafting in cycling; in contrast to shorter distances, drafting is not allowed in Half-Ironman and longer distances. It is well known that cycling before running leads to an alteration in running performance (Bernard et al., 2003). Nevertheless, Ofoghi et al. (2016) demonstrated that triathletes, who were the fastest in swimming and cycling, were also the fastest in overall race time at the Olympic distance.

Our results, however, are different to findings for shorter triathlon distances such as the Olympic distance triathlon where the cycling split did not affect the subsequent running efficiency (Cala et al., 2009). The cycling split performance seems to have a different influence on overall race time considering the length of the triathlon race. In the Olympic distance triathlon, running ( $47 \%)$ and then cycling $(\sim 36 \%)$ had the greatest contribution, whereas for the Ironman distance, cycling $(\sim 40 \%)$ and running $(\sim 40 \%)$ showed similar contributions (Figueiredo et al., 2016).

The relatively fastest runners were the fastest in overall race performance

The fourth main observation was that athletes in Double Iron ultra-triathlons showed relatively the fastest running times, whereas athletes in Deca Iron ultra-triathlons had the slowest relative running times. Besides, the fastest performance group was relatively the fastest in running. It has been found previously that running performance is more important to overall performance for Double and Triple Iron ultratriathlons than to Deca Iron ultra-triathlons (Lepers et al., 2011). Among Triple Iron ultratriathletes, running was more important than cycling and swimming (Knechtle et al., 2007; Knechtle and Kohler, 2009).

An improved running performance after cycling can be explained in short distance triathlons by drafting. Practicing drafting allows triathletes to save energy during cycling and improve running, and is more beneficial for faster than slower athletes (Hausswirth et al., 1999). In long-distance triathlons such as Ironman triathlon and longer distances, drafting is forbidden and, therefore, athletes cannot profit from following closely other athletes.

Non-finishers were not relatively slower than finishers in swimming and cycling

Another important finding was that the split times in swimming and cycling for nonfinishers were not relatively slower than for finishers. There is no data available regarding at which split each athlete stopped; however, it is most likely that the non-finishers broke down in the running split since it has been shown that the running split is the most important one in an ultra- 
triathlon (Hausswirth et al., 1999; Knechtle and Kohler, 2009).

Pacing differences between the sexes

The above mentioned patterns of pacing in relation to ultra-triathlon versions and performance groups were observed for both women and men; however, the relationship magnitudes were weaker for women. This variation in magnitude might be partially attributed to the relatively small number of women participating in such events, resulting in consistently larger variations in split and race times. In 'Ultraman Hawaii', a multi-stage ultratriathlon covering two Ironman distances within three days, women were observed to pace differently than men. For the fastest women, performance decreased on day 1 , but was maintained on days 2 and 3. For the fastest men, performance worsened on days 1 and 2, but improved on day 3 (Knechtle and Nikolaidis, 2016).

\section{Competition density was higher for men and in the short distance versions}

The last important finding was that men were more competitive, i.e. there were more competitors per unit of time (minute) than women, especially in the short ultra-distance races. This might be due to differences in motivation between female and male triathletes (Houston et al., 2011; López-Fernández et al., 2014; Stoeber et al., 2009).

\section{Limitations and strengths}

A limitation of the study was the relatively small number of participants in the long versions of ultra-triathlons, especially of women. Unfortunately, there was no available data on age and experience in order to insert more variables in our analyses. A strength of the present study is its novelty, since it is the first research, to the best of our knowledge, conducted on pacing strategies in ultra-triathlons longer than the Ironman distance.

Practical applications for athletes and coaches

These findings have important practical applications for ultra-triathletes and coaches working with them, especially regarding pacing. The fastest finishers in a shorter race such as Double Iron ultra-triathlon had relatively slow swimming and cycling times and fast running times. Thus, ultra-triathletes should consider this information to optimize their own pacing strategy. A practical application for athletes competing in a Double Iron ultra-triathlon is to save energy during swimming and cycling in order to have enough energy while running. Another piece of information of practical value concerned the differences in pacing among different ultratriathlon distances. Longer versions presented relatively faster swimming (especially) and cycling split times, and slower running times, suggesting that triathletes should adapt their pacing strategy according to the ultra-triathlon distance in which they participate. A practical application for athletes intending to compete in a Quintuple or Deca Iron ultra-triathlon is to consider smarter pacing in swimming and cycling in order to perform well in the running split. The overall fastest competitors in the longer version ultra-triathlons, compared with the shorter version ultra-triathletes, spent less time swimming and more time running. This might reflect that this very small group of athletes competing in the longest ultra-triathlon distances has not yet learned how to appropriately pace in such a long event.

\section{Conclusions}

In summary, a higher percentage of athletes finished longer races. The relatively fastest swimmers and the fastest cyclists were the slowest in overall race time in longer races, whereas the fastest runners achieved the best overall race times in shorter races. Athletes competing in a Double Iron ultra-triathlon need to consider conserving energy during swimming and cycling for the running split, and athletes intending to compete in a Quintuple or Deca Iron ultra-triathlon need to consider smarter pacing in swimming and cycling splits in order to perform well in the running split.

\section{Acknowledgements}

We thank Patricia Villiger for her contribution to the English editing. 


\section{References}

Abbiss CR, Laursen PB. Describing and understanding pacing strategies during athletic competition. Sports Med, 2008; 38: 239-252

Angehrn N, Rust CA, Nikolaidis PT, Rosemann T, Knechtle B. Positive pacing in elite Ironman triathletes. Chin J Physiol, 2016; 59: 305-314

Baron B, Moullan F, Deruelle F, Noakes TD. The role of emotions on pacing strategies and performance in middle and long duration sport events. Br J Sports Med, 2011; 45: 511-517

Bernard T, Hausswirth C, Le Meur Y, Bignet F, Dorel S, Brisswalter J. Distribution of power output during the cycling stage of a triathlon world cup. Med Sci Sports Exerc, 2009; 41: 1296-1302

Bernard T, Vercruyssen F, Grego F, Hausswirth C, Lepers R, Vallier JM, Brisswalter J. Effect of cycling cadence on subsequent $3 \mathrm{~km}$ running performance in well trained triathletes. Br J Sports Med, 2003; 37: 154-158

Cala A, Veiga S, García A, Navarro E. Previous cycling does not affect running efficiency during a triathlon World Cup competition. J Sports Med Phys Fitness, 2009; 49: 152-158

Delextrat A, Tricot V, Bernard T, Vercruyssen F, Hausswirth C, Brisswalter J. Drafting during swimming improves efficiency during subsequent cycling. Med Sci Sports Exerc, 2003; 35: 1612-1619

Edwards A, Polman R. Pacing in sport and exercise: A psychophysiological perspective. Hauppauge, NY: Nova Publishers; 2012

Figueiredo P, Marques EA, Lepers R. Changes in Contributions of Swimming, Cycling, and Running Performances on Overall Triathlon Performance Over a 26-Year Period. J Strength Cond Res, 2016; 30: 2406-2415

Foster C, Hoyos J, Earnest C, Lucia A. Regulation of energy expenditure during prolonged athletic competition. Med Sci Sports Exerc, 2005; 37: 670-675

Foster C, Snyder AC, Thompson NN, Green MA, Foley M, Schrager M. Effect of pacing strategy on cycle time trial performance. Med Sci Sports Exerc, 1993; 25: 383-388

Hausswirth C, Lehénaff D, Dréano P, Savonen K. Effects of cycling alone or in a sheltered position on subsequent running performance during a triathlon. Med Sci Sports Exerc, 1999; 31: 599-604

Herbst L, Knechtle B, Lopez CL, Andonie JL, Fraire OS, Kohler G, Rüst CA, Rosemann T. Pacing strategy and change in body composition during a Deca iron triathlon. Chin J Physiol, 2011; 54: 255-263

Houston M, Dolan S, Martin S. The impact of physical, nutritional, and mental preparation on triathlon performance. J Sports Med Phys Fitness, 2011; 51: 583-594

Knechtle B, Duff B, Amtmann G, Kohler G. Cycling and running performance, not anthropometric factors, are associated with race performance in a triple iron triathlon. Res Sports Med, 2007; 15: 257-269

Knechtle B, Knechtle P, Lepers R. Participation and performance trends in ultra-triathlons from 1985 to 2009. Scand J Med Sci Sports, 2011; 21: e82-90

Knechtle B, Kohler G. Running performance, not anthropometric factors, is associated with race success in a Triple Iron Triathlon. Br J Sports Med, 2009; 43: 437-441

Knechtle B, Nikolaidis PT. Sex differences in pacing during 'Ultraman Hawaii'. PeerJ, 2016; 2016

Knechtle B, Rosemann T, Lepers R, Rüst CA. A comparison of performance of Deca Iron and Triple Deca Iron ultra-triathletes. SpringerPlus, 2014; 3: 461

Le Meur Y, Bernard T, Dorel S, Abbiss CR, Honnorat G, Brisswalter J, Hausswirth C. Relationships between triathlon performance and pacing strategy during the run in an international competition. Int J Sports Physiol Perform, 2011; 6: 183-194

Le Meur Y, Hausswirth C, Dorel S, Bignet F, Brisswalter J, Bernard T. Influence of gender on pacing adopted by elite triathletes during a competition. Eur J Appl Physiol, 2009; 106: 535-545

Lepers R, Knechtle B, Knechtle P, Rosemann T. Analysis of ultra-triathlon performances. Open Access J Sports Med, 2011; 2: 131-136

(c) Editorial Committee of Journal of Human Kinetics 
López-Fernández I, Merino-Marbán R, Fernández-Rodríguez E. Examining the relationship between sex and motivation in triathletes. Percept Motor Skills, 2014; 119: 42-49

Ofoghi B, Zeleznikow J, Macmahon C, Rehula J, Dwyer DB. Performance analysis and prediction in triathlon. J Sports Sci, 2016; 34: 607-612

Peeling P, Landers G. Swimming intensity during triathlon: A review of current research and strategies to enhance race performance. J Sports Sci, 2009; 27: 1079-1085

Peeling PD, Bishop DJ, Landers GJ. Effect of swimming intensity on subsequent cycling and overall triathlon performance. Br J Sports Med, 2005; 39: 960-964

Stoeber J, Uphill MA, Hotham S. Predicting race performance in triathlon: The role of perfectionism, achievement goals, and personal goal setting. J Sport Exerc Psychol, 2009; 31: 211-245

Vleck VE, Bürgi A, Bentley DJ. The consequences of swim, cycle, and run performance on overall result in elite olympic distance triathlon. Int J Sports Med, 2006; 27: 43-48

Vleck VE, Bentley DJ, Millet GP, Bürgi A. Pacing during an elite Olympic distance triathlon: Comparison between male and female competitors. J Sci Med Sport, 2008; 11: 424-432

Wu SS, Peiffer JJ, Peeling P, Brisswalter J, Lau WY, Nosaka K, Abbiss CR. Positive swim pacing improves sprint triathlon performance in trained athletes. Int J Sports Physiol Perform, 2016; 11: 1024-1028

Wu SSX, Peiffer JJ, Brisswalter J, Nosaka K, Lau WY, Abbiss CR. Pacing strategies during the swim, cycle and run disciplines of sprint, Olympic and half-Ironman triathlons. Eur J Appl Physiol, 2015; 115: 1147-1154

\section{Corresponding author:}

\section{Prof. Dr. med. Beat Knechtle}

Facharzt FMH für Allgemeinmedizin

Gesundheitszentrum St. Gallen

Vadianstrasse 26

9001 St. Gallen

Switzerland

Telefon $+41(0) 712269300$

Telefax $+41(0) 712269301$

E-Mail beat.knechtle@hispeed.ch 